\title{
Immunohistochemical Study of CDK5 and Ki-67 in Cervical Precancerous Lesion and Carcinoma
}

\author{
Amira Nasr Elsokary*, Samah Mohamed Attiah, Bahaa Bedair Ghannam \\ Department of Pathology, Faculty of Medicine, Al-Azhar University, Egypt \\ *Corresponding author: Amira Nasr Elsokary, Tel: 002-01003997007, Email: ameranasr.medg@azhar.edu.eg
}

\begin{abstract}
Background: cervical carcinoma is one of the commonest female tumors worldwide arises from cervical intraepithelial neoplasia. Cervical cancer is a multifactorial process and infection with some types of human papilloma virus (HPV) has been suggested as the most important risk factor. The genesis of cancer was correlated with abnormal regulation of DNA damage and repair. A research proved that CDK5 was mandatory for the DNA damage response in cancer cells. Ki-67 is a nuclear protein that is associated with RNA transcription and cell cycle progression.

Aim of the work: the purpose of this study was to evaluate the expression of CDK5 in cervical precancerous lesions and carcinoma to clarify its role in carcinogenesis and progression of cervical cancer, as well as the expression of Ki-67.

Material and Method: specimens included (55 cases) consisted of precancerous lesions (15), SCC (30), adenocarcinoma (10). All specimens were formalin-fixed and embedded in paraffin blocks. Multiple serial 5-microne thick sections were cut from the paraffin blocks of the specimens; one was stained by Hematoxylin and Eosin for histopathological examination, Other Sections were immunostained with antibodies to CDK5 and K-i67.

Results: high tumor grade and high FIGO stage were positively correlated with CDK5 IHC expression (P-value $=0.012$ and 0.042) and with Ki67 IHC expression (p-value $=0.017$ and 0.044 ) respectively.

Conclusion: there is a direct positive correlation of both CDK5 and Ki-67 expression with high tumor grade and high FIGO stage of cervical carcinoma.
\end{abstract}

Keywords: precancerous, SCC, adenocarcinoma, CDK5, Ki-67

\section{Introduction}

Cervical cancer is usually associated with human papilloma virus (HPV) ${ }^{(\mathbf{1})}$. Cervical intraepithelial neoplasia (CIN) is the precursor lesions of cervical squamous cell carcinoma ${ }^{(2)}$. CIN 1 Known as mild dysplasia; CIN 2 Known as moderate dysplasia and CIN 3 known as severe dysplasia. The grading of CIN depends upon the proportion of the epithelium occupied by basaloid atypical cells reflecting a progressive loss of maturation and increasing lesion severity ${ }^{(3)}$.

The World Health Organization (WHO) (4) recognizes three basic types of invasive carcinoma of the cervix: squamous cell carcinoma, adenocarcinoma, and other epithelial tumors.

Histological grading divides Squamous cell carcinoma (SCC) into three subtypes, depends upon the proportion of the tumor keratinization with the formation of keratin pearls and mitoses, Well differentiated (grade 1), Moderately differentiated (grade 2), Poorly differentiated (grade 3$)^{(\mathbf{5})}$.
Grading of cervical adenocarcinoma depends upon gland architecture (glandular and papillary architecture versus solid areas) and its nuclear features ${ }^{\left({ }^{(}\right)}$. The staging system for tumors of the cervix is International Federation of Gynecologists and Obstetricians (FIGO) ${ }^{(7)}$.

The genesis of cancer was related to abnormal mechanism of DNA damage and repair. Cyclin dependant kinase 5 (CDK5) is important for the DNA damage response in tumor cells ${ }^{(8)}$. Ki-67 is a prognostic factor for the assessment of biopsies from precancerous and cervical cancer. Clinically; Ki-67 has been shown to correlate with the stage of tumors ${ }^{(9)}$.

\begin{abstract}
Aim of the work
The purpose of this study is to evaluate the expression of CDK5 in cervical precancerous lesions and carcinoma to clarify its role in carcinogenesis and progression of cervical cancer, as well as the expression of Ki-67. CDK5 reactivity and proliferation rate as
\end{abstract}


assessed by Ki-67 was correlated with the clinicopathological parameters

\section{Materials and Methods}

This study included total 55 cervical specimens (32 punch biopsy and 23 total abdominal hysterectomy cases) of formalin fixed paraffin embedded blocks. The blocks collected from the archival files of the Histopathology department, AL-Zahraa University hospital and some private laboratories during the period from January 2016 to May 2018. Clinicopathological information was obtained from medical charts after obtaining an informed consent and approval of Local Ethical committee. Three serial 5-microne thick sections were cut from the paraffin blocks of the specimens; one was stained by Hematoxylin and Eosin to evaluate the diagnosis and to access the histopathological staging and grading of the tumors. While the other sections were mounted on positive charged slides and immunostained by rabbit polyclonal antibody against CDK5 and rabbit monoclonal antibody against Ki-67. Cervical cancers were classified according to WHO 2014 Classification of cervical Tumors (4). Tumor stage was evaluated according to FIGO staging system ${ }^{(7)}$. Grading of cervical SCC was done according to Alexandra et al. ${ }^{(5)}$. Grading of cervical adenocarcinoma was done according to Diaz et al. ${ }^{\left({ }^{6}\right)}$.

For CDK5 immunostaining, staining of brain tissue was used as a positive control (10). For K-i67 immunostaining, staining of breast cancer tissue was used as a positive control ${ }^{(11)}$. As a negative control for two markers, a tumor tissue was processed through the above sequences but the primary antibody was omitted, instead phosphate buffer solution was added. The whole sections were scanned and evaluated at 10x magnification of the light microscope. Positive staining of CDK5 was indicated as yellowish to brown cytoplasmic or nucleocytoplasmic colour of the tumor cells while positive staining for Ki-67 was indicated as brown staining of the nucleus of tumor cells. CDK5 immunostaining was scored according to the staining intensity as follows: 0 (no staining), 1(weak staining, light yellow), 2 (moderate staining, yellow brown) and 3 (strong staining, brown) ${ }^{(\mathbf{1 2})}$.

$\mathrm{Ki}-67$ was defined as the percentage of $\mathrm{Ki}-$ 67 positive cells, the staining score was classified into: Negative (0): staining is observed in less than 5\%tumor cells. Weak $(1+)$ : staining is detected in $5 \%-30 \%$ of the tumor cells. Moderate (2+): staining is detected in $30 \%-50 \%$ of the tumor cells. Strong (3+): staining is detected in more than $50 \%$ of the tumor cells ${ }^{(\mathbf{1 3})}$.

\section{Statistical analysis}

Data management and analysis were performed using statistical analysis Systems. Data were summarized using means and standard deviations. Categorical data were summarized as percentages. A one-way analysis of variance (ANOVA) was used when comparing between more than two means. ANOVA refers to the number of independent variables in analysis of Variance test. One-way has one independent variable with 2 levels. Chisquare (x2) test of significance was used in order to compare proportions between two qualitative parameters. The p-value was considered significant as the following: Probability (P-value)

- P-value <0.05 was considered significant.

- P-value <0.001 was considered as highly significant.

- P-value >0.05 was considered insignificant.

\section{Results}

The age of patients ranged from 34 to 68 years, with a mean age of $49.4 \pm 9.11 \mathrm{SD}$. The studied cases consisted of precancerous lesions (15/55 cases) represented $27.3 \%$ and carcinoma cases (40/55 cases) [SCC 30, adenocarcinoma 10] represented $72.7 \%$. The grade of carcinoma cases was: grade I ( 9 cases), grade II ( 21 cases) and grade III (10 cases). Tumor stage of carcinoma cases as following: (3 cases) were diagnosed as stage IA, (13 cases) as stage IB and (5 cases) as stage II Table (1). 
Table (1): Clinical data of patients included in this study.

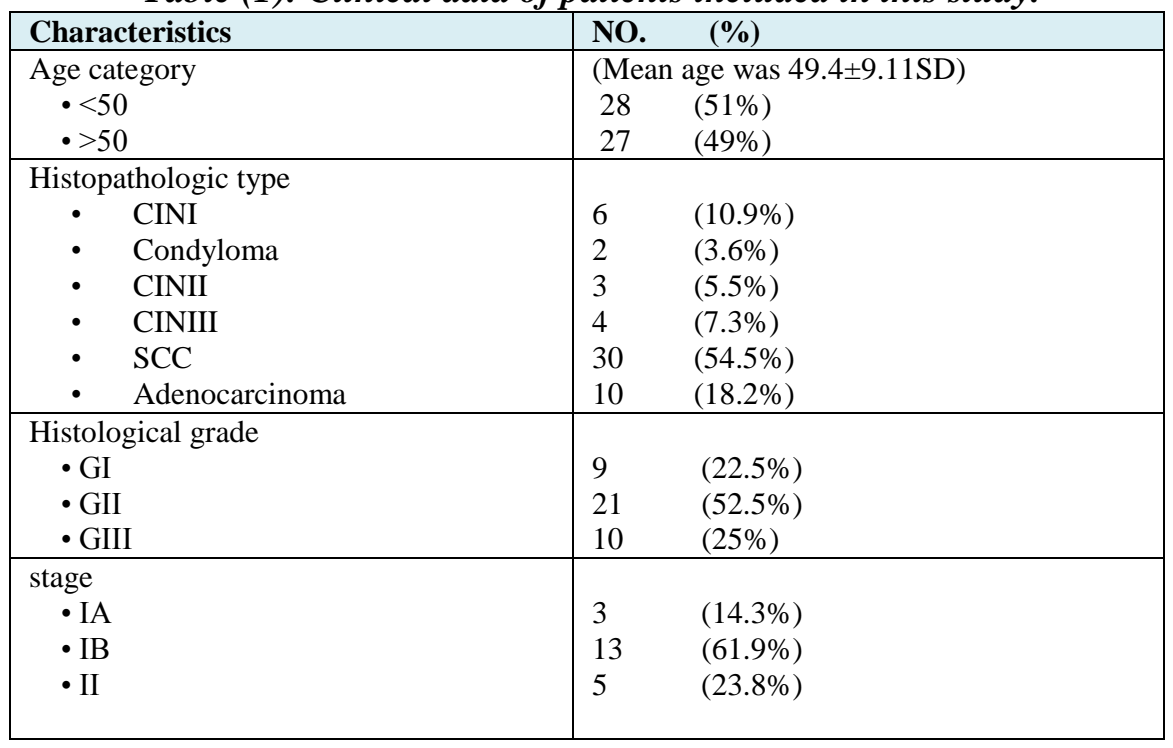

In our study 50/55 cases (90.9\%) showed positive CDK5 expression and 5/55 cases $(9.1 \%)$ were negative (Fig. $1 \boldsymbol{D}, \boldsymbol{E}$ and $\boldsymbol{F}$ ). CDK5 expression showed statistically significant relation with histopathological type $\left(\mathrm{p}\right.$-value $\left.=0.003^{*}\right)$. CDK5 expression showed statistically significant relation with high tumor grade $\left(\mathrm{p}\right.$-value $\left.=0.012^{*}\right)$.CDK5 expression showed statistically significant relation with high tumor stage ( $\mathrm{p}$-value $=0.042^{*}$ ) Table (2).

Table (2): Clinicopathological association of CDK5 expression in cervical precancerous lesion and carcinoma

\begin{tabular}{|c|c|c|c|c|c|c|}
\hline \multicolumn{2}{|c|}{ Clinico Pathological Parameter } & \multicolumn{5}{|c|}{ CDK5 Expression } \\
\hline Characteristics & NO. $\quad(\%)$ & $0(\%)$ & $1+(\%)$ & $2+(\%)$ & $3+(\%)$ & p-value \\
\hline $\begin{array}{cl}\text { Histopathologic type } \\
: & \text { CINI } \\
: & \text { Condyloma } \\
: & \text { CINII } \\
: & \text { CINIII } \\
: & \text { SCC } \\
\cdot & \text { Adenocarcinoma }\end{array}$ & $\begin{array}{ll}6 & (10.9) \\
2 & (3.6) \\
3 & (5.5) \\
4 & (7.3) \\
30 & (54.5) \\
10 & (18.2)\end{array}$ & $\begin{array}{l}2(33.3) \\
0(0.0) \\
0(0.0) \\
0(0.0) \\
3(10) \\
0(0.0)\end{array}$ & $\begin{array}{l}4(66.7) \\
1(50) \\
0(0.0) \\
0(0.0) \\
6(20) \\
0(0.0)\end{array}$ & $\begin{array}{l}0(0.0) \\
1(50) \\
3(100) \\
0(0.0) \\
9(30) \\
3(30)\end{array}$ & $\begin{array}{l}0(0.0) \\
0(0.0) \\
0(0.0) \\
4(100) \\
12(40) \\
7(70)\end{array}$ & $0.003 *$ \\
\hline $\begin{array}{l}\text { Histological grade } \\
- \text { GI } \\
\bullet \text { GII } \\
\cdot \text { GIII } \\
\end{array}$ & $\begin{array}{l}(22.5) \\
(52.5) \\
(25)\end{array}$ & $\begin{array}{l}1(33.3) \\
2(66.7) \\
0(0.0)\end{array}$ & $\begin{array}{l}2(33.3) \\
4(66.7) \\
0(0.0)\end{array}$ & $\begin{array}{l}6(50) \\
5(41.7) \\
1(8.3) \\
\end{array}$ & $\begin{array}{l}0(0.0) \\
10(52.6) \\
9(47.4)\end{array}$ & $0.012 *$ \\
\hline $\begin{array}{l}\text { Stage } \\
\cdot \text { IA } \\
\cdot \text { IB } \\
\cdot \text { II }\end{array}$ & $\begin{array}{ll}3 & \\
(14.3) & \\
13 & (61.9) \\
5 & (23.8)\end{array}$ & $\begin{array}{l}2(66.7) \\
1(33.3) \\
0(0.0)\end{array}$ & $\begin{array}{l}0(0.0) \\
5(83.3) \\
1(16.7)\end{array}$ & $\begin{array}{l}1(16.7) \\
4(66.7) \\
1(16.7)\end{array}$ & $\begin{array}{l}0(0.0) \\
3(50) \\
3(50)\end{array}$ & $0.042 *$ \\
\hline
\end{tabular}

In our study, 53 cases (96.3\%) showed positive Ki-67 expression and 2 cases (3.6\%) were negative (Fig. 1 G, $\boldsymbol{H}$ and I). Ki67 expression showed highly statistically significant relation with histopathological type (p-value $\left.<0.001^{* *}\right)$. Ki-67 expression showed statistically significant relation with high tumor grade $(\mathrm{p}$-value $=0.017 *)$.

Ki-67 expression showed statistically significant relation with high tumor stage $\left(\mathrm{p}\right.$-value $\left.=0.044^{*}\right)$ Table (3).

Table (3): Clinicopathological association of Ki67 expression in cervical precancerous lesion and carcinoma 


\begin{tabular}{|c|c|c|c|c|c|c|c|c|}
\hline \multicolumn{2}{|c|}{ Characteristics } & NO. & $(\%)$ & $0(\%)$ & $1+(\%)$ & $2+(\%)$ & $3+(\%)$ & p-value \\
\hline $\begin{array}{r}\text { Histopa } \\
\text { • } \\
\text { • } \\
\text { • } \\
\text { • }\end{array}$ & $\begin{array}{l}\text { gic type } \\
\text { CINI } \\
\text { Condyloma } \\
\text { CINII } \\
\text { CINIII } \\
\text { SCC } \\
\text { Adenocarcinoma }\end{array}$ & $\begin{array}{c}6 \\
2 \\
3 \\
4 \\
30 \\
10\end{array}$ & $\begin{array}{l}(10.9) \\
(3.6) \\
(5.5) \\
(7.3) \\
(54.5) \\
(18.2)\end{array}$ & $\begin{array}{l}2(33.3) \\
0(0.0) \\
0(0.0) \\
0(0.0) \\
0(0.0) \\
0(0.0)\end{array}$ & $\begin{array}{l}3(50) \\
2(100) \\
0(0.0) \\
0(0.0) \\
0(0.0) \\
0(0.0)\end{array}$ & $\begin{array}{l}1(16.7) \\
0(0.0) \\
3(100) \\
0(0.0) \\
15(50) \\
4(40)\end{array}$ & $\begin{array}{l}0(0.0) \\
0(0.0) \\
0(0.0) \\
4(100) \\
15(50) \\
6(60)\end{array}$ & $<0.001 * *$ \\
\hline $\begin{array}{l}\text { Histolog } \\
\cdot \text { - GI } \\
\cdot \text { GII } \\
\cdot \text { - GIII }\end{array}$ & rade & $\begin{array}{l}9 \\
21 \\
10\end{array}$ & $\begin{array}{l}(22.5) \\
(52.5) \\
(25)\end{array}$ & $\begin{array}{l}0(0.0) \\
0(0.0) \\
0(0.0)\end{array}$ & $\begin{array}{l}0(0.0) \\
0(0.0) \\
0(0.0)\end{array}$ & $\begin{array}{l}9(47.4) \\
10(52.6) \\
0(0.0)\end{array}$ & $\begin{array}{l}0(0.0) \\
11(52.4) \\
10(47.6)\end{array}$ & $0.017 *$ \\
\hline $\begin{array}{r}\text { Stage } \\
\cdot \text { I } \\
\cdot \text { Il } \\
\cdot \text { I }\end{array}$ & & $\begin{array}{l}3 \\
13 \\
5\end{array}$ & $\begin{array}{l}(14.3) \\
(61.9) \\
(23.8)\end{array}$ & $\begin{array}{l}0(0.0) \\
0(0.0) \\
0(0.0)\end{array}$ & $\begin{array}{l}0(0.0) \\
0(0.0) \\
0(0.0)\end{array}$ & $\begin{array}{l}3(18.8) \\
11(68.8) \\
2(12.5)\end{array}$ & $\begin{array}{l}0(0.0) \\
2(40) \\
3(60)\end{array}$ & $0.044 *$ \\
\hline
\end{tabular}

Correlation between CDK5 and Ki-67 expression in our total 55 cases showed direct positive relationship and highly significant statistical correlation between CDK5 and Ki-67 (P value $<0.001 * *$ ) Table (4). Figure 2.

Table (4): Correlation between CDK5 and Ki-67 in the studied cases

\begin{tabular}{|c|c|c|c|c|c|c|c|c|}
\hline \multirow{2}{*}{\multicolumn{2}{|c|}{ Ki67 }} & \multicolumn{4}{|c|}{ CDK5 } & \multirow{2}{*}{ Total } & \multicolumn{2}{|c|}{ Chi-square test } \\
\hline & & 0 & +1 & +2 & +3 & & $\mathbf{x} 2$ & p-value \\
\hline \multirow{2}{*}{0} & No. & 2 & 0 & 0 & 0 & 2 & \multirow{10}{*}{67.803} & \multirow{10}{*}{$<0.001 * *$} \\
\hline & $\%$ & $40.0 \%$ & $0.0 \%$ & $0.0 \%$ & $0.0 \%$ & $3.6 \%$ & & \\
\hline \multirow{2}{*}{+1} & No. & 0 & 4 & 1 & 0 & 5 & & \\
\hline & $\%$ & $0.0 \%$ & $36.4 \%$ & $6.3 \%$ & $0.0 \%$ & $9.1 \%$ & & \\
\hline \multirow{2}{*}{+2} & No. & 3 & 7 & 12 & 1 & 23 & & \\
\hline & $\%$ & $60.0 \%$ & $63.6 \%$ & $75.0 \%$ & $4.3 \%$ & $41.8 \%$ & & \\
\hline \multirow{2}{*}{+3} & No. & 0 & 0 & 3 & 22 & 25 & & \\
\hline & $\%$ & $0.0 \%$ & $0.0 \%$ & $18.8 \%$ & $95.7 \%$ & $45.5 \%$ & & \\
\hline \multirow{2}{*}{ Total } & No. & 5 & 11 & 16 & 23 & 55 & & \\
\hline & $\%$ & $100.0 \%$ & $100.0 \%$ & $100.0 \%$ & $100.0 \%$ & $100.0 \%$ & & \\
\hline
\end{tabular}

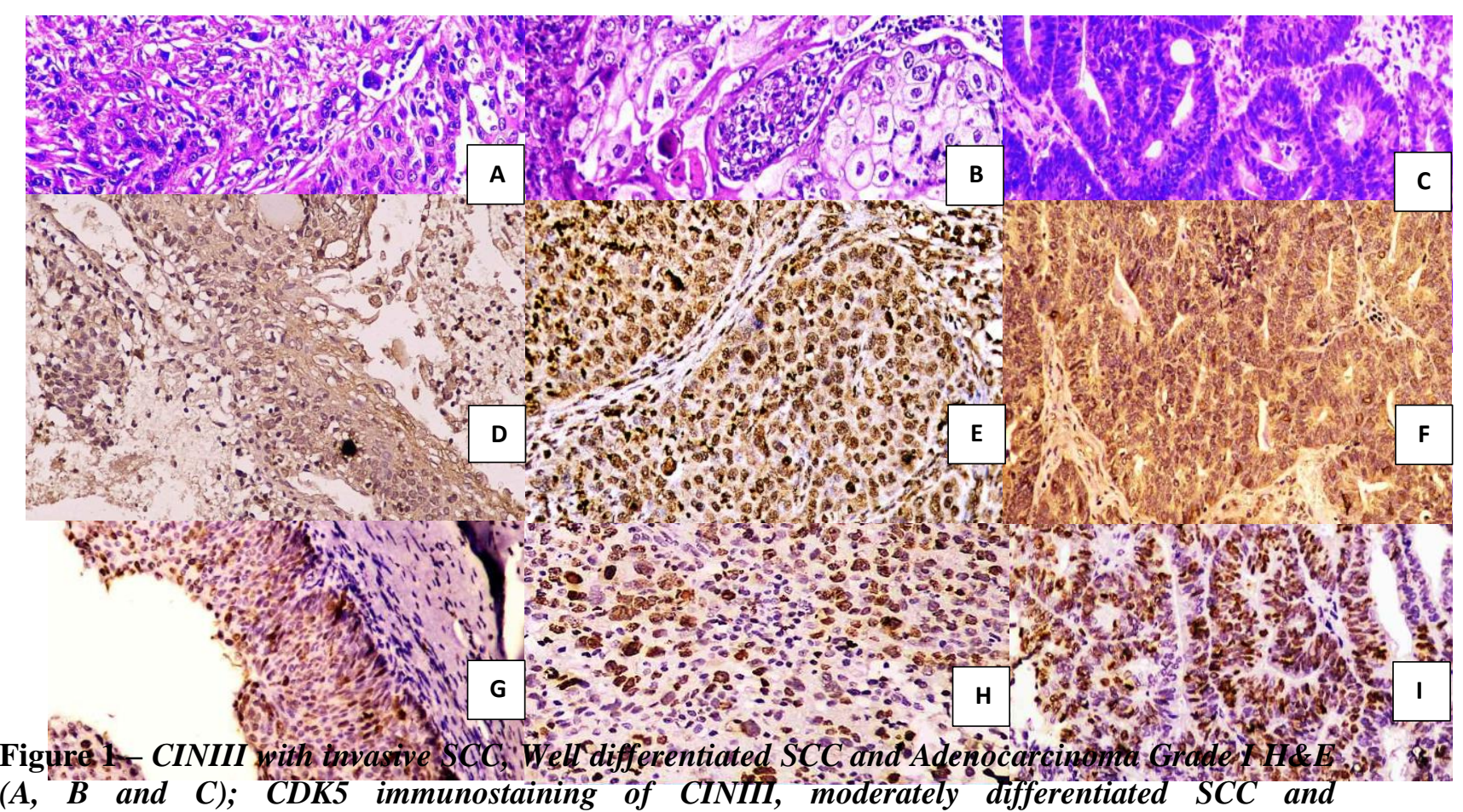




\section{Adenocarcinoma Grade I (D, E and F); Ki-67 immunostaining of CINIII, poorly differentiated SCC and Adenocarcinoma Grade II (G, $H$ and I).}

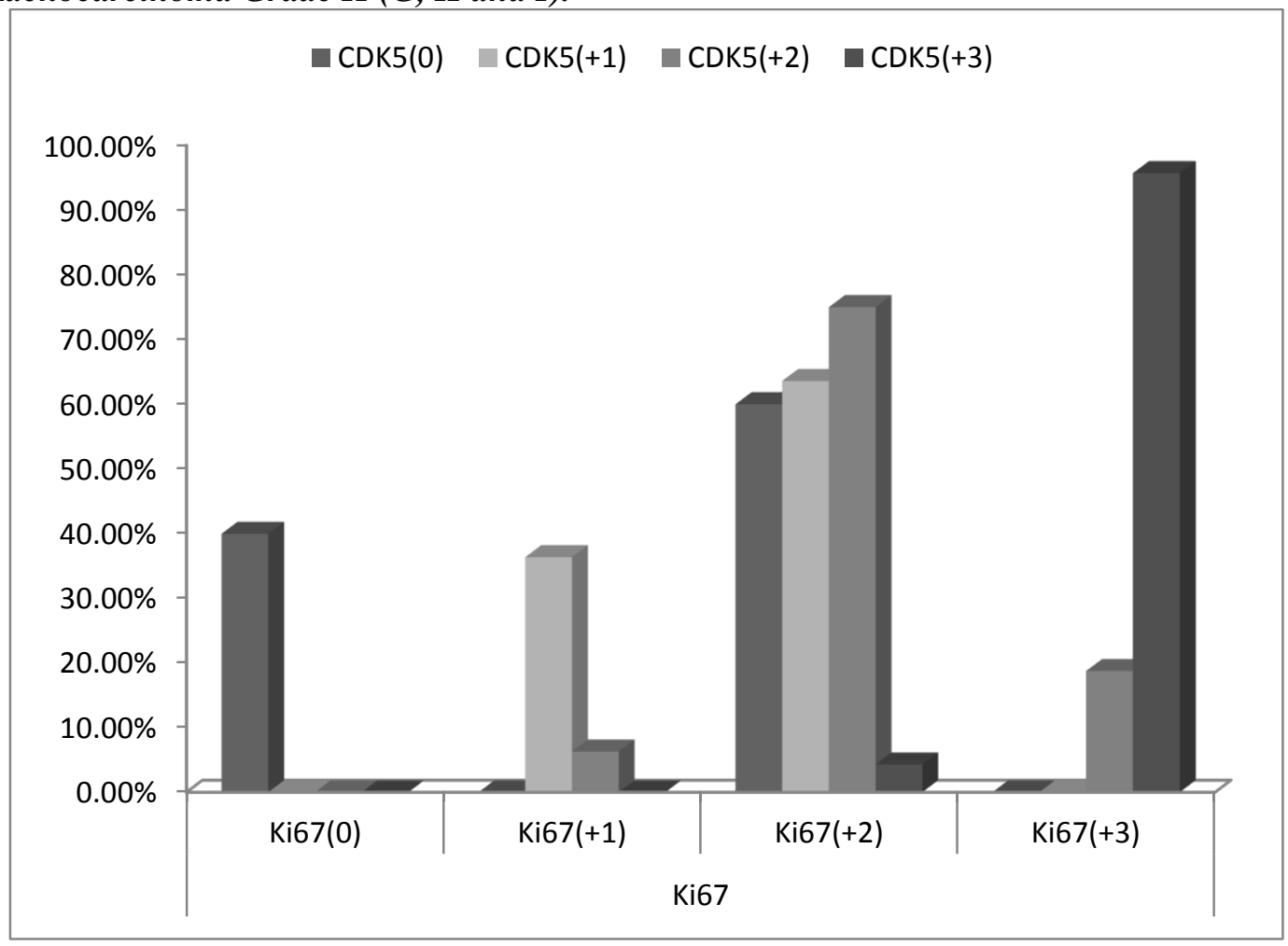

Figure 2: Correlation between Ki67 and CDK5 in the studied cases.

\section{Discussion}

In our study precancerous cervical cases represented $27.3 \%$ of all cases. The most common precancerous lesions were CINI representing $40 \%$ followed by CINII representing $20 \%$ followed by CINIII representing $26.6 \%$ of precancerous cases. The least common one was Condyloma accuminatum representing $13.3 \%$ of precancerous cases. This result is near to the result of Melodi et al. ${ }^{(\mathbf{1 4})}$ who revealed that in their study precancerous lesions were $24 \%$. In our study cervical cancer cases represented $75 \%$ of all cases. As regard histopathological tumor types; SCC was the most common malignant tumor subtype in our study representing $75 \%$ followed by adenocarcinoma representing $25 \%$ of carcinoma cases. Our findings are in agreement with Deng-Hua et al. (10) who reported that $73 \%$ of their cases were SCC, $18 \%$ were adenocarcinoma of all cases. According to WHO, squamous cell carcinoma of the cervix: accounts for the vast majority (80$90 \%$ ) of cases, adenocarcinoma of the cervix is rarer than SCC (5-20\%) ${ }^{(4)}$.

As regarding the grade of tumor, the majority of our studied cases were grade II $(52.5 \%)$, followed by grade III $(25 \%)$ while grade I (22.5\%) was the least cases. This finding is in agreement with those of Torre et al. ${ }^{(15)}$ and Siegel et al. ${ }^{(16)}$ who found that grade II was the most frequent and represented $52 \%$ and $51 \%$ of their total cases respectively. As regard tumor FIGO stage (2014), 3 cases (14.3\%) were stage IA, 13 cases $(61.9 \%)$ were stage IB and 5 (23.8\%) cases were II stage.

Immunohistochemical evaluation of CDK5 and Ki67 expression were used to assess their potential role in the tumor carcinogenesis and progression of cervical cancer.

The immune expression of CDK5 was positive in 50 cases $(90.9 \%)$ and negative in 5cases $(9.1 \%)$. In Precancerous lesions CDK5 expression was low in CINI represented $(66.7 \%)$ and highest in CINIII represented (100\%).

Regarding histopathologic types of carcinoma in our study CDK5 expression level was high in cervical adenocarcinoma (100\%) followed by SCC tissues $(90 \%)$ and higher in cancer cervix compared with precancerous lesions $(23.7 \%, 67.3 \%)(\mathrm{P}$ value $=0.003 *)$ indicating that CDK5 up-regulation may be relative to the carcinogenesis and progression of cervical cancer. 
Our finding is higher than the frequency found by Deng-Hua et al. ${ }^{(10)}$ who reported that positive rates of CDK5 were 50, 58.8, and $62.7 \%$ in CIN, adenocarcinoma and SCC, respectively. These differences may be attributed to the variation in distribution of histopathologic types as well as grading, staging and scoring systems used.

Regarding pathological grade of carcinoma cases of our study CDK5 expression was higher in grade II $(52.5 \%)$ than grade III $(25 \%)$ and grade $\mathrm{I}(22.5 \%)(\mathrm{P}$ value $=0.012 *)$ indicating that CDK5 expression is higher in high grades of the tumors.

These results differ from Deng-Hua et al. (10) who reported that CDK5 positive expression level with pathological grade in all cancer cases were III $(75.9 \%)$ was higher than pathological grade I (42.9\%) and pathological grade II $(51.0 \%)(\mathrm{P}$ value $<0.001)$ These differences may be attributed to the variation in distribution of histopathologic types as well as grading, staging and scoring systems used.

Regarding FIGO stage higher CDK5 expression was found to be correlated with advanced FIGO stage, in stage IA CDK5 expression was $(14.3 \%)$, in stage IB CDK5 expression was $(61.9 \%)$ and in stage II CDK5 expression was $(23.8 \%)(\mathrm{P}$ value $=0.042 *)$ These results indicated that CDK5 could be a potential predictor for the deterioration and development of cervical cancer.

Based on our observations, we revealed that the up-regulated expression of CDK5 from precancerous lesions to cervical cancer tissues might be correlated with the development of cervical carcinoma from high grade squamous intraepithelial lesion.

In our study, 53 cases (96.3\%) showed positive Ki-67 expression and 2 cases (3.6\%) showed negative $\mathrm{Ki}-67$ expression In precancerous lesions; we observed an increasing proportion of Ki-67 positive subjects with increasing histologic severity, $(66.7 \%)$ in CINI cases, $(100 \%, 100 \%)$ in CINII, and CINIII cases.

Regarding histopathologic types of our study Ki67 proliferation rate was higher (strong $3+$ ) in adenocarcinoma (70\%) than SCC $(40 \%)$ and higher in cancer than $\mathrm{CIN}(100 \%, 23.6 \%)$ (P value $<0.001^{* *}$ ). This finding was near to the results of Ankitha and Venkataramappa ${ }^{(13)}$ who reported strong positive expression (3+) of Ki67 in adenocarcinoma, SCC and CIN III 83\%, $67 \%$ and $50 \%$ respectively.
Regarding pathological grade of carcinoma cases of our study Ki-67 expression was higher in grade II $(52.5 \%)$ than grade III $(25 \%)$ and grade I $(22.5 \%)\left(\mathrm{P}\right.$ value $=0.017^{*}$

) indicating that CDK5 expression is higher in high grades of the tumors.

Regarding FIGO stage higher ki-67 proliferation rate was found to be correlated with advanced FIGO stage, in stage IA Ki-67 expression was (14.3\%), in stage IB Ki67 expression was $(61.9 \%)$ and in stage II K-i67 expression was (23.8) ( $\mathrm{P}$ value $<0.044 *)$.

The present study showed there is a direct positive relationship and highly significant statistical correlation between CDK5 and Ki-67 in total 55 cases (P value $<0.001 * *$ ). These findings suggest that the detection of both CDK5 and ki-67 expression would be complementary for predicting tumor aggressiveness, prognosis and treatment of cervical cancer patients.

\section{Conclusion}

We conclude that Progression of cervical cancer based on carcinogenesis can be assessed by CDK5 and uncontrolled cell proliferation evaluated by $\mathrm{Ki}-67$. $\mathrm{Ki}-$ 67 and CDK5 markers are recommended as complementary tests for differentiating between cervical precancerous lesions and carcinoma.

\section{References}

1. Léonard B, Kridelka $F$ and Delbecque (2016): A Clinical and Pathological Overview of Vulvar Condyloma Acuminatum, Intraepithelial Neoplasia, and Squamous Cell Carcinoma. BioMed Research International, $8: 1-11$.

2. Ingkapairoj $N$, Luanratanakorn $S$ and Chumworathayi B (2013): Incidences of cervical intraepithelial neoplasia 2-3 or cancer pathologic diagnoses in patients with a high grade squamous intraepithelial lesion Pap smear attending a colposcopy clinic at Srinagarind hospital. Asian Pac J Cancer, 13:6203-6.

3. He Y, Wu YM and Zhao Q (2015): Clinical analysis of patients underwent hysterectomy for stage I cervical cancer or high grade cervical intraepithelial neoplasia. Zhonghua $\mathrm{Fu}$ Chan Ke Za Zhi, 50: 516-21.

4. Kurman RJ, Carcangiu ML, Herrington CS and Young RH (2014): WHO/IARC Classification of Tumours, 4th Edition, Volume 6. publications.iarc.fr/Book-And-ReportSeries/Who-Iarc-Classification-Of-Tumours 
5. Alexandra NK, Farnaz $D$ and Teri AL (2016): Protocol for the Examination of Specimens from Patients with Carcinoma of the Uterine

Cervix. www.cap.org/ShowProperty?nodePath=/UCM Con/.../pdf/cp-cervix-16protocol

6. Diaz A, Roma AA, Park KJ and Rasty G (2013): Invasive endocervical adenocarcinoma: proposal for a new pattern-based classification system with significant clinical implications: a multi-institutional study. Int $\mathbf{J}$ Gynecol Pathology, 32:592-601.

7. Pecorelli $S$, Zigliani $L$ and Odicino $F$ (2009): Revised FIGO staging for carcinoma of the cervix. Int J Gynaecol Obstetric,105:107-8.

8. Shupp A, Casimiro MC and Pestell RG (2017): Biological functions of CDK5 and potential CDK5 targeted clinical treatments. Oncotarget, 8:17373-17382.

9. Fatemeh SA, Akbar S and Masoumeh $P$ (2013): Evaluation of Ki67, p16 and CK17 Markers in Differentiating Cervical Intraepithelial Neoplasia and Benign Lesions" Iran J Med Science, 38: 15-21.

10. Deng-Hua $P$, Mei-Lin $Z$, Xiao-Miao $L$ and Xing-Gu L (2016): Evaluation and clinical significance of cyclin-dependent kinase 5 expression in cervical lesions: a clinical research study in Guangxi, China. Eur J Med Res., 21: 28-30.

11. Eskander RN and Tewari KS (2014): Beyond angiogenesis blockade: targeted therapy for advanced cervical cancer. J Gynecol Oncol., 25:249-59.

12. Yu QS, Jian WX, and ChaoH Z (2016): Low Expression of CDK5 and p27 Are Associated with Poor Prognosis. J Cancer, 7: 1049-1056.

13. Ankitha $H$ and Venkataramappa $S$ (2017): Role of p16/INK4a and Ki-67 as specific biomarkers for cervical intraepithelial neoplasia: An institutional study, 9: 104-110.

14. Melodi $M$, Gerd $B$ and Heinrich $H$ (2015): CIN III lesions and regression: retrospective analysis of 635 cases. BMC Infect Disease, 15: 541-2.
15. Torre LA, Bray F and Ferlay J (2016): Global cancer statistics. CA Cancer J Clin., 65:87-108.

16. Siegel RL, Miller KD, Jemal A (2017): Cancer statistics, CA Cancer J Clin., 67: 7-30.

\section{Introduction}

Cervical cancer is usually associated with human papilloma virus (HPV) ${ }^{(\mathbf{1})}$. Cervical intraepithelial neoplasia (CIN) is the precursor lesions of cervical squamous cell carcinoma ${ }^{(2)}$. CIN 1 Known as mild dysplasia; CIN 2 Known as moderate dysplasia and CIN 3 known as severe dysplasia. The grading of CIN depends upon the proportion of the epithelium occupied by basaloid atypical cells reflecting a progressive loss of maturation and increasing lesion severity ${ }^{(3)}$.

The World Health Organization (WHO) (4) recognizes three basic types of invasive carcinoma of the cervix: squamous cell carcinoma, adenocarcinoma, and other epithelial tumors.

Histological grading divides Squamous cell carcinoma (SCC) into three subtypes, depends upon the proportion of the tumor keratinization with the formation of keratin pearls and mitoses, Well differentiated (grade 1), Moderately differentiated (grade 2), Poorly differentiated (grade 3$)^{(\mathbf{5})}$.

Grading of cervical adenocarcinoma depends upon gland architecture (glandular and papillary architecture versus solid areas) and its nuclear features ${ }^{\left({ }^{(6)}\right.}$. The staging system for tumors of the cervix is International Federation of Gynecologists and Obstetricians (FIGO) ${ }^{(7)}$.

The genesis of cancer was related to abnormal mechanism of DNA damage and repair. Cyclin dependant kinase 5 (CDK5) is important for the DNA damage response in tumor cells ${ }^{(8)}$. Ki-67 is a prognostic factor for the assessment of biopsies from precancerous and cervical cancer. Clinically; Ki-67 has been shown to correlate with the stage of tumors ${ }^{(9)}$. 